\title{
INSERCIÓN PROFESIONAL. PROGRAMA DE ACOMPAÑAMIENTO DOCENTES NOVELES DEL CONSEJO DE FORMACIÓN EN EDUCACIÓN (CFE)
}

\author{
Soledad Rodríguez Rodríguez \\ soledadrodriguezcerp@,gmail.com \\ https://orcid.org/0000-0001-7292-2512 \\ Centro Regional de Profesores del Litoral \\ Salto, Uruguay \\ José Pablo Márquez \\ pabloelde195@gmail.com \\ https://orcid.org/0000-0001-8909-0353 \\ Centro Regional de Profesores del Litoral \\ Salto, Uruguay
}

Recibido: 01/11/2020 Aceptado: 01/12/2020

\begin{abstract}
Resumen
Hace ya una década que en nuestro país se vienen desarrollando investigaciones y programas vinculados a la temática de la inserción profesional de los noveles docentes. Las primeras experiencias laborales y su relación con la definición de la identidad profesional han cobrado especial relevancia a la luz de una nueva perspectiva en lo referente a la formación del profesorado, que no se agota en las instancias iniciales, sino que se concibe con un carácter permanente y transversal a las trayectorias laborales. En el año 2010 fue puesto en marcha el "Proyecto Acompañamiento a docentes noveles del Uruguay", enmarcado en las políticas educativas del Consejo de Formación en Educación (CFE) y con el auspicio de la oficina uruguaya de la Organización de Estados Iberoamericanos (OEI). Esta iniciativa, que pretendía mejorar los procesos de socialización institucional de los docentes principiantes y propiciar su efectiva integración a las diversas comunidades educativas en las que desarrollarían su actividad profesional, ha crecido y diversificado sus acciones, procurando al día de hoy, abarcar a un mayor número de actores del sistema de educación pública. A través de este artículo se pretende dar a conocer una experiencia innovadora, que pone el foco en los primeros años de actividad laboral, con el fin de acompañar a aquellos que comienzan a construir su identidad profesional en las aulas uruguayas.
\end{abstract}

Palabras clave: formación docente- inserción profesional- novel docente- formación permanente - trayectorias educativas

\section{INSERÇÃO PROFISSIONAL. PROGRAMA DE ACOMPANHAMENTO DE PROFESSORES INICIANTES DO CONSELHO DE TREINAMENTO DE EDUCAÇÃO}

(CFE)

\section{Resumo}

Há uma década, pesquisas e programas relacionados ao tema da integração profissional de novos professores vêm se desenvolvendo em nosso país. As primeiras experiências de trabalho e sua relação com a definição de identidade profissional ganharam relevância especial à luz de uma nova perspectiva sobre a formação de professores, que não se esgota nos estágios iniciais, mas é concebida com um caráter permanentes e transversais às trajetórias de trabalho. Em 2010, foi 
lançado o "Projeto de Acompanhamento de Novos Professores no Uruguai", enquadrado nas políticas educacionais do Conselho de Formação Educacional (CFE) e sob os auspícios do escritório uruguaio da Organização dos Estados Ibero-Americanos (OEI). Essa iniciativa, que teve como objetivo melhorar os processos de socialização institucional dos professores iniciantes e promover sua efetiva integração nas diversas comunidades educacionais nas quais exerceria sua atividade profissional, tem crescido e diversificado suas ações, buscando hoje abranger um maior número de atores no sistema público de ensino. Este artigo tem como objetivo apresentar uma experiência inovadora focada nos primeiros anos de atividade profissional, a fim de acompanhar aqueles que começam a construir sua identidade profissional nas salas de aula uruguaias.

Palavras-chaves: formação de professores - integração profissional - novo professoreducação permanente- trajetórias educativas

\title{
PROFESSIONAL INSERTION. EDUCATION TRAINING COUNCIL (CFE) NOVICE TEACHERS ACCOMPANIMENT PROGRAM
}

\begin{abstract}
For a decade now, research and programs related to the subject of professional integration of new teachers have been developing in our country. The first work experiences and their relationship with the definition of professional identity have gained special relevance in light of a new perspective regarding teacher training, which is not exhausted in the initial stages, but is conceived with a character permanent and transversal to work trajectories. In 2010, the "Project for Accompanying New Teachers in Uruguay" was launched, framed within the educational policies of the Education Training Council (CFE) and under the auspices of the Uruguayan office of the Organization of Ibero-American in 2010, the "Project for Accompanying New Teachers in Uruguay" was launched, framed within the educational policies of the Education Training Council (CFE) and under the auspices of the Uruguayan office of the Organization of Ibero-American States. This initiative, which aimed to improve the institutional socialization processes of beginning teachers and promote their effective integration into the various educational communities in which they would carry out their professional activity, has grown and diversified its actions, seeking to date, to encompass a greater number of actors in the public education system. The aim of this article is to present an innovative experience, which focuses on the first years of working activity, in order to accompany those who begin to build professional identity in Uruguayan classrooms.
\end{abstract}

Key Words: teacher training- professional insertion- new teacher- permanent educationeducationals paths

\section{Introducción}

El intercambio académico desarrollado en 2019 entre docentes del Cerp del Litoral y de diversas universidades brasileñas (UFOP-PUCSP- Unicid-UFSCar) generó la oportunidad de conocer distintas tradiciones y experiencias en la formación de docentes de ambos países. En tal sentido, en la ventana abierta al diálogo, en el ir al encuentro del otro, fue posible redescubrir y reflexionar acerca de la propia realidad institucional y académica, incluso vivencial, en lo que refiere a la incorporación, año a año, de los nuevos colegas al ámbito educativo, que también lo 
es laboral. Esta situación ha sido entendida en nuestro país, desde el marco institucional y sistémico, como una fase importante en la conformación de la identidad docente. Al incorporarse al ámbito escolar en tanto y en cuanto este último como comunidad educativa, el recién egresado pasa por un proceso de adaptaciones y reacomodos no simplemente pasivos, de incorporación y reinterpretación de símbolos y ritos comunitarios, que se torna necesario conocer y poner en comunicación con los demás.

Desde el plano institucional, como decíamos, se ha llevado adelante en Uruguay el Programa de Acompañamiento de Docentes Noveles del CFE, bajo el entendido de que es necesario acompañar, precisamente, a los docentes novatos y/o con no más de tres años de experiencia laboral, a fin de acompañarlos como sujetos en la construcción de su propia identidad profesional. El objetivo de este artículo es analizar desde lo documental el trayecto desplegado por el citado Programa, así como realizar un balance de sus logros y perspectivas.

\section{Inserción profesional y formación permanente}

Una de las principales líneas de trabajo desarrolladas en los últimos años por el Consejo de Formación en Educación (CFE) ha sido la de la formación permanente de los docentes y educadores sociales uruguayos. En este sentido, el foco se ha puesto en los noveles, es decir, aquellos profesionales de la educación que se encuentran en la etapa inicial de su carrera (hasta 5 años) y que ejercen su labor en algunas de las instituciones educativas bajo la órbita de la Administración Nacional de Educación Pública (ANEP).

Como señalan múltiples investigadores (Bolívar, 2007, Carlos Marcelo, 2009, Alen, 2009), los primeros años de ejercicio profesional son claves en la formación de la identidad docente y en el desarrollo de buenas prácticas educativas. Es durante este período que tiene lugar la incorporación efectiva a los contextos y niveles para los cuales estos docentes y educadores se han formado. Así, a través de la práctica se construyen experiencias personales y académicas que moldean las formas de hacer y vivenciar la docencia, por lo que estos años se consideran también años de formación y aprendizaje.

Se produce el inicio de una nueva etapa profesional:

La obtención del título habilita para ejercer. En ese sentido, se cierra el tiempo de estudiante y se abre el tiempo de la profesionalidad docente, pero los procesos formativos no terminan con ello. Se inaugura ahí el comienzo de los procesos orientados al desarrollo profesional. (MORAES,2011, p. 7) 
Desde esta perspectiva, la formación docente no finaliza con la formación de grado, sino que se prolonga a lo largo de la trayectoria profesional de los individuos, adquiriendo un carácter continuo y permanente. Esto implica para el Consejo de Formación en Educación, asumir un rol más amplio como institución formadora, desarrollando dispositivos de profesionalización continua y diseñando proyectos de acompañamiento profesional.

En una primera instancia, se consideró prioritario ofrecer a los noveles docentes las herramientas necesarias para hacer frente a las demandas y desafíos propios de las primeras experiencias laborales, no siempre previstas o contempladas en su formación inicial. Se pretendían minimizar las incertidumbres propias de esta etapa, utilizando el acompañamiento de los nuevos docentes como una herramienta de mejora educativa, fortaleciendo el vínculo entre las instituciones formadoras y sus egresados, actuando en el pasaje de la formación de grado a las primeras inserciones laborales.

El espacio escolar constituye un ámbito de formación por excelencia, así que, rápidamente se hizo evidente que, más allá del desarrollo profesional de estos docentes, la interacción entre colegas con diferentes trayectorias y biografías, resultaba mutuamente enriquecedora, generándose ámbitos de intercambio significativo para todos los actores involucrados.

Por tanto, el acompañamiento a los noveles docentes y educadores no puede limitarse a su inserción en una determinada cultura escolar, sino que debe comprenderse como una oportunidad para la indagación, la reflexión y la innovación educativa.

Como plantea Beatriz Alen, “el acompañamiento a los principiantes necesita revisar y sistematizar dispositivos específicos y diferenciados de aquellos típicos de la formación inicial" (Alen, B.,2017, p.17). El punto de partida para esta autora radica en la necesidad de considerar al docente principiante como un sujeto en proceso de construir su experticia profesional, y no como un profesional con déficit de formación.

\section{Programa de Acompañamiento Docentes Noveles del CFE}

En su artículo 1, la Ley General de Educación (LGE) No 18437, promulgada en el año 2008, reconoce a la educación como un derecho humano fundamental, y en ese sentido, el Estado asume como garante y promotor de la formación de todos sus habitantes, "a lo largo de toda la vida, facilitando la continuidad educativa" (LGE, 2008, p.1). Esta nueva perspectiva que 
supone, además, apostar a la extensión de una educación de calidad, implica pensar en la formación integral del individuo, contemplando necesidades, contextos e intereses diversos y particulares. Propone un cambio de paradigma, puesto que se asume que el conocimiento está en permanente expansión y que, en consecuencia, la formación del sujeto es un proceso siempre inacabado. Para el Estado, y en particular para la educación pública uruguaya, genera nuevas exigencias, al demandar del sistema educativo acciones y recursos tendientes a efectivizar dicha declaración.

En el caso puntual de la Formación Docente, desde hace algunos años, se ha comenzado a transitar hacia una política de Desarrollo Profesional Docente. A través del documento Orientaciones y Objetivos del Consejo de Formación en Educación 2015-2020 (Res 53 Acta 15 CFE 15 de mayo de 2015) se realiza un balance de las actuaciones desarrolladas hasta el momento y se trazan líneas a futuro, acordes a las necesidades del sistema y a las perspectivas teóricas a las que adhiere el Consejo. Éste concibe el Desarrollo Profesional Docente "como la formación inicial y continua de los profesionales de la educación (maestros, profesores, maestros técnicos, educadores sociales y otras formaciones que la educación nacional requiera) desde una perspectiva integradora" (p.1)

Se pretende generar las condiciones para que los docentes en ejercicio accedan a espacios y oportunidades para producir nuevos conocimientos y para la adquisición de herramientas y capacidades que le permitan desempeñarse con niveles de excelencia.

Para cumplir con estas orientaciones, contando con el apoyo de la Organización de Estados Iberoamericanos (OEI) y los aportes teóricos de destacados referentes de la región, se puso en marcha el Proyecto Docentes Noveles del Uruguay. Éste tiene por objetivo:

contribuir a la inserción del novel profesional de la educación en procesos de innovación educativa, para generar dispositivos de acompañamiento que contribuyan a su integración en la comunidad educativa, fomentando la reflexión y análisis en y desde las prácticas cotidianas (novelesuruguay.cfe.edu.uy)

El programa, destinado al acompañamiento de los noveles docentes, incluye la formación de tutores y mentores, la generación de dispositivos y estrategias que hagan factible dicho acompañamiento, así como la articulación entre los diversos centros de formación docente y de enseñanza media involucrados en el proceso. 
Este proyecto toma como referente a Marta Souto (1999), quien define los dispositivos de formación como un conjunto de acciones, acepción que en una primera instancia parecería ser sencilla y acotada, pero que en realidad implica en cambio, una serie de supuestos mucho más complejos sobre la búsqueda del saber, el desarrollo de ciertas capacidades profesionales y la posibilidad de reflexión, individual y colectiva, que llevan al sujeto a situaciones de profundo análisis personal y laboral. Sumado a esto, Laura Gaidulewicz, (1999), de cuyos postulados también se nutre el proyecto, afirma que el concepto de dispositivo debe remitirnos a Foucault, y a el atributo que éste reconoce en él, es decir su "...capacidad productora de sujeciones y sentidos, que se construyen y re construyen de manera permanente, inacabada, en un juego de orden- desorden" (Gaiduewicz, L.1999, p.73)

Desde esta perspectiva el dispositivo permite generar cambios, conscientes y provocados, tanto a nivel individual como social, más allá de la posibilidad de que tengan lugar otras transformaciones, sorpresivas e inesperadas, pero igualmente movilizadoras. Situados en el campo de la educación, el dispositivo se vuele entonces un espacio estratégico para la comunidad docente. Pensar el dispositivo en clave pedagógica, supone abrir las puertas al aprendizaje y la formación.

Como consta en el documento marco que da origen al Programa, se entiende que las estrategias de acompañamiento deben consistir

En un trabajo formativo sustentado en la reflexión sobre el quehacer profesional en el cual se implican los docentes que acompañan y los principiantes en un plano de horizontalidad ya que se trata de vínculo entre colegas, que excede la transmisión de información, estimulando procesos de crecimiento profesional individual y colaborativos.

\section{Etapas iniciales} (DOCUMENTO NOVELES DOCENTES, 2015, p.3)

Las primeras acciones para la implementación de este proyecto se remontan a los años 2010-2011, cuando, con carácter de experiencia piloto, se comenzó a trabajar con 14 centros de formación docente. En los años siguientes se amplió la cantidad de instituciones y docentes involucrados, procurándose la formación de recursos humanos para el acompañamiento de los noveles profesionales.

A partir de 2012 el énfasis fue puesto en la formación de mentores formadores y en la elaboración de múltiples dispositivos de acompañamiento, como medio para ampliar y 
profundizar las acciones de protección a las trayectorias profesionales en todos los niveles formativos.

Hacia 2014 se conformó un equipo de trabajo, integrado por cuatro directoras y una profesora de centros educativos del CFE, que se abocó a la planificación y realización de diversas actividades, tanto de carácter nacional como regional. Los dispositivos desarrollados pretendían estimular procesos de crecimiento profesional tanto individuales como colectivos, a partir del trabajo colaborativo de expertos y principiantes.

Progresivamente fueron integrados también como beneficiarios del proyecto los estudiantes del último año de la carrera de Magisterio, Profesorado y Educador Social. Desde la División de Planeamiento Educativo del CFE, en el año 2016, se realizó un relevamiento tendiente a identificar y comprender las necesidades de los noveles docentes y educadores del Uruguay. Este estudio y otros existentes en el país demostraron que sus principales preocupaciones no se relacionaban a cuestiones disciplinares, sino a la acción pedagógica más general. La atención a la diversidad, la inclusión, el ejercicio de la labor docente en contextos desfavorables, las dificultades de aprendizaje, la incorporación de tecnología en el aula, fueron algunas de las inquietudes más frecuentes. Asimismo, en ellos se expresa que tanto para los docentes noveles como para los expertos que están en contacto con ellos, la formación inicial recibida debería complementarse con una formación permanente orientada a promover la reflexión sobre la profesión docente y fomentar el trabajo colaborativo. Esta información fue tomada como insumo para el diseño de nuevas propuestas incluidas en el programa.

\section{Organización actual}

Actualmente, es a la Coordinación General a la que le corresponde llevar a cabo la articulación interinstitucional a fin de promover la inserción de Noveles docentes en procesos de innovación educativa. Se encarga, además, de diseñar, organizar y promover actividades de extensión, de formación permanente y de la coordinación de diversos equipos de trabajo derivados de la aplicación del proyecto.

Por su parte, el equipo articulador, es el responsable de acompañar en territorio a los diversos actores involucrados en esta iniciativa. Entre sus cometidos se encuentra el de identificar las necesidades de éstos con respecto a la formación profesional permanente, gestionando cursos, ya sea en modalidad presencial o virtual. Es responsable de actuar como 
nexo, entre los Mentores del CFE y los Tutores del CES (Consejo de Educación Secundaria), del CETP (Consejo de Educación Técnico Profesional) y Educadores sociales. En este sentido, se ocupa de incentivar el diseño e implementación de proyectos de acompañamiento a los noveles profesionales de la educación, generando, además, ámbitos de reflexión colectiva acerca de las prácticas docentes.

El acompañamiento pedagógico a los noveles es realizado por los Mentores, es decir, formadores que se desempeñan en los diferentes Centros e Institutos dependientes del CFE. Aunque no es un requisito excluyente para participar de esta iniciativa, se prioriza a quienes desde el año 2017 hayan aprobado el Curso semipresencial de desarrollo profesional para el acompañamiento a docentes y educadores noveles. Para la efectiva aplicación de los proyectos elaborados por estos Mentores, se prevé que las horas de trabajo que éste insume, puedan ser consideradas como horas de Departamento, previa presentación del mismo a la Coordinación General y a las Direcciones de las instituciones involucradas.

El rol de Tutor, con funciones de inducción de los noveles en el ámbito laboral más inmediato, es desempeñado por docentes de Enseñanza Media. El CES dispuso que fueran el Profesor Orientador Pedagógico (POP) quien asumiera como figura de acompañamiento de los noveles docentes en el centro educativo, dado que entre sus cometidos se encuentra el mejoramiento de las prácticas de enseñanza, de la calidad de los aprendizajes y la convivencia institucional. A estos cometidos se suman el de promover la formación continua del personal docente y la autoevaluación del centro referida a la implementación del correspondiente proyecto de acompañamiento profesional. El Consejo de Formación Técnico Profesional consideró que esta tarea debía ser ejercida por el Maestro/Profesor Técnico, por su doble formación, teórica y práctica y la experiencia adquirida, tanto en lo referido a lo pedagógico, como a lo científico.

Los tutores convocan y coordinan acciones en el centro educativo, poniendo en contacto a noveles docentes, docentes expertos, mentores del CFE y equipos de dirección institucional. Se espera que faciliten la inserción del novel docente poniéndolo en conocimiento de las particularidades del ámbito en el que se desempeñan, así como a través de la orientación ante distintas situaciones, ya sea el manejo de conflictos, la atención a la diversidad de los estudiantes $\mathrm{u}$ otros aspectos del quehacer docente. Otro factor que se considera relevante es el de guiar al novel docente en el reconocimiento de los pormenores de la cultura institucional del centro en 
cuestión. Por todo lo anterior, se consideró pertinente adjudicar 5 horas semanales mensuales para el cumplimiento de estas actividades y a diferencia del caso anterior, se estableció como requisito indispensable para ejercer la tutoría, el haber realizado y aprobado el Curso de Tutores de Acompañamiento a Noveles Docentes.

Por último, también integra el Proyecto un Grupo de Apoyo inter- institucional, conformado por representantes de la Dirección de Planeamiento Educativo del CFE, el CES y el CETP, la Dirección General del CFE, las Direcciones de los Centros del CFE, la Asamblea Técnico Profesional del CFE, la carrera de Educador Social, Coordinación General del Proyecto y representantes de la OEI.

\section{Actividades recientes}

En el marco de este proyecto se han realizado múltiples actividades, ya sea en territorio o a través de la modalidad virtual, con espíritu descentralizador y el objetivo de abarcar a un número cada vez mayor de participantes.

- Ciclos de Encuentros, articulados con el CES y el CETP, contando con la participación de tutores, noveles docentes y orientadores del programa. Se desarrollan a partir de la identificación de aquellos temas sobre los que los noveles mencionan dudas e incertidumbres, permitiendo ámbitos para su problematización y reflexión.

- Primer Encuentro de Formación, destinado a noveles, tutores, mentores, Directores de Centros Educativos del CES, CETP, CFE y Educadores Sociales.

- Encuentros Regionales de Formación, con el propósito de brindar herramientas teóricoprácticas, fomentar espacios de reflexión para noveles educadores y fortalecer la construcción del rol de tutores del Proyecto en Educación Media y de docentes mentores del CFE.

- Acompañamiento virtual a los actores involucrados.

- Visitas periódicas de la coordinación y el equipo articulador a centros de formación en educación y de educación media.

Como resultado, se han generado experiencias individuales y colectivas, que por su potencial formativo, han sido recogidas en distintas publicaciones coordinadas por los equipos de trabajo del programa de acompañamiento a noveles docentes. Las reflexiones en torno a la 
práctica educativa, a la identidad docente, así como el intercambio de distintas teorías sobre la necesidad de acompañamiento a los docentes principiantes, impulsaron el desarrollo de las narraciones docentes y les permitió a los protagonistas relatar en primera persona sus vivencias e impresiones.

\section{Balance y valoración del proyecto}

En el transcurso del año 2019 se llevaron adelante proyectos que involucraron treinta (30) tutores de los centros de enseñanza media, así como se han implementado diez (10) proyectos que comprenden a treinta y cuatro (34) mentores del CFE, alcanzando a más de setecientos (700) noveles que alcanzan a los distintos niveles de la enseñanza pública.

La propuesta, caracterizada por la extensión académica y el trabajo colaborativo, ha tenido como objetivos tanto las recopilación y conceptualización de experiencias de acompañamiento a noveles docentes, como la creación de un "banco de recursos" para quienes se involucran en la continuidad del proyecto.

Se han establecido vínculos tanto a nivel regional como internacional, nutriendo a estas iniciativas de múltiples miradas, permitiendo que el conocimiento generado sobre el tema en el exterior, potencie las investigaciones locales, generando una potente línea de indagación sobre la realidad de los noveles docentes en el Uruguay.

\section{Consideraciones finales}

La experiencia adquirida en el marco de este proyecto ha permitido retroalimentar las actividades y espacios de participación que se han ido generando a lo largo del tiempo. De esta forma, se han realizado ajustes y modificaciones tendientes a la adaptación a las necesidades de las nuevas generaciones de egresados y noveles docentes. Por otra parte, en base a las narrativas generadas no sólo por parte de los profesores principiantes, sino también de tutores y mentores, ha permitido constatar en numerosos casos la importancia del diálogo inter- generacional. Ello ha redundado en un fortalecimiento del sentido de comunidad, especialmente por el trasiego de conocimiento generado no simplemente a partir del vínculo cotidiano, sino fundamentalmente a través del reconocimiento de fines comunes y vivencias compartidas. El novel docente descubre en su par "veterano" caminos ya transitados, que pueden ser reutilizados al lidiar con sus propias inseguridades, así como el más experiente reperfila su rol docente y se nutre del entusiasmo juvenil. 
La inserción profesional de los nuevos docentes debe ser abordada como una cuestión colectiva e institucional, como un proceso que a través de las tramas generadas por los vínculos intergeneracionales potencie a todos los sujetos involucrados en él. La importancia de un proyecto como el descripto radica en que todo el proceso de construcción de vínculos de una comunidad educativa se teje de manera consciente, en forma sistemática, alentando dinámicas de retroalimentación teórico- prácticas a partir del colectivo educativo. Todo lo señalado no implica mitigar los probables conflictos y sinergias producto de la interacción entre educadores de distintas generaciones, sino que desde el propio sistema educativo se aliente a que cada comunidad desarrolle un método, una hoja de ruta autónoma para resolver sus problemas.

\section{Referencias bibliográficas}

Alen, B. (2009) El acompañamiento a los maestros y profesores en su primer puesto de trabajo en Revista Profesorado. Vol. 13. $\mathrm{N}^{\circ} 1$. pp.79-87. Recuperado de: https://www.ugr.es/ recfpro/rev131ART5.pdf

Bolívar, A. (2007) La formación inicial del profesorado de secundaria y su identidad profesional en Estudios Sobre Educación N${ }^{\circ}$ 12. Universidad de Navarra. pp.13-30. Recuperado de https://revistas.unav.edu/index.php/estudios-sobreeducacion/article/download/24326/20409

Marcelo García, C. (Coord.). (2009) El profesorado principiante: Inserción a la docencia. Barcelona: Octaedro.

Documento Noveles Docente Expediente $N^{\circ}$ 2018-25-5-00009. Recuperado de: https://www.ces.edu.uy/files/Proyectos_Docentes_Noveles_de_Uruguay.pdf

Duglio. I. ,Nizarala,P., Olivera, R. y otros (2011) Experiencias de Acompañamiento de noveles docentes en Uruguay. Recuperado de: https://oei.org.uy/uploads/files/programs/1/projects/20/expuru1.pdf

Ley de General de Educación N 18437. Publicada D.O. 16 ene/009 - № 27654 Recuperado de: https://www.ineed.edu.uy/images/pdf/-18437-ley-general-de-educacion.pdf

Salle, C., Nossar, K. (comp) (2017) La potencialidad de las interacciones entre educadores expertos y noveles. Aportes teóricos, dispositivos de trabajo y producciones. Montevideo: Planeamiento Educativo CFE.

Souto, M. et al. (1999) Grupos y dispositivos de formación. Buenos Aires: Universidad de Buenos Aires 
Duglio. I. ,Nizarala,P., Olivera, R. y otros (2011) Experiencias de Acompañamiento de noveles docentes en Uruguay. Recuperado de:

https://oei.org.uy/uploads/files/programs/1/projects/20/expuru1.pdf

Orientaciones y Objetivos. Consejo de Formación en Educación 2015-2020. Recuperado de http://www.cfe.edu.uy/images/stories/pdfs/documentos_aprobados_cfe/documento_ori entaciones objetivos.pdf

http://novelesuruguay.cfe.edu.uy/ 